\title{
Role of 3D Alumina Foam Support on the Formation and Dispersion of Active NiMoS Phase for Hydrodesulfurization Application
}

Mustapha Umar ${ }^{\mathrm{a}}$, Abdulkadir Tanimu ${ }^{\mathrm{a}}$, Saheed A. Ganiyu ${ }^{\mathrm{a}}$, Hatem Dafalla ${ }^{\mathrm{b}}$, Ivan Kozhevnikov ${ }^{\mathrm{c}}$, Khalid Alhooshani ${ }^{\mathrm{a} *}$

${ }^{a}$ Department of Chemistry, King Fahd University of Petroleum and Minerals, Dhahran 31261, Saudi Arabia.

${ }^{\mathrm{b}}$ Center for Engineering Research (CER), King Fahd University of Petroleum and Minerals, Dhahran 31261, Saudi Arabia

${ }^{c}$ Department of Chemistry, University of Liverpool, Liverpool, L69 7ZD, United Kingdom.

*Corresponding author

Email: hooshani@kfupm.edu.sa

Phone: +966138603065

Fax: +966 138604277 


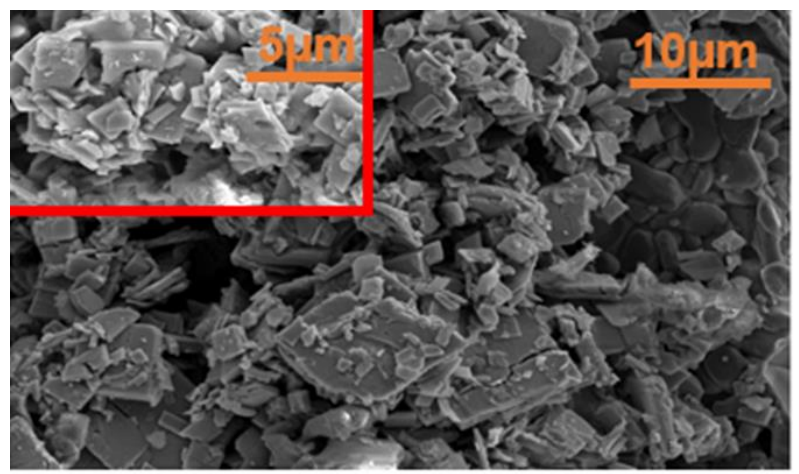

(a)

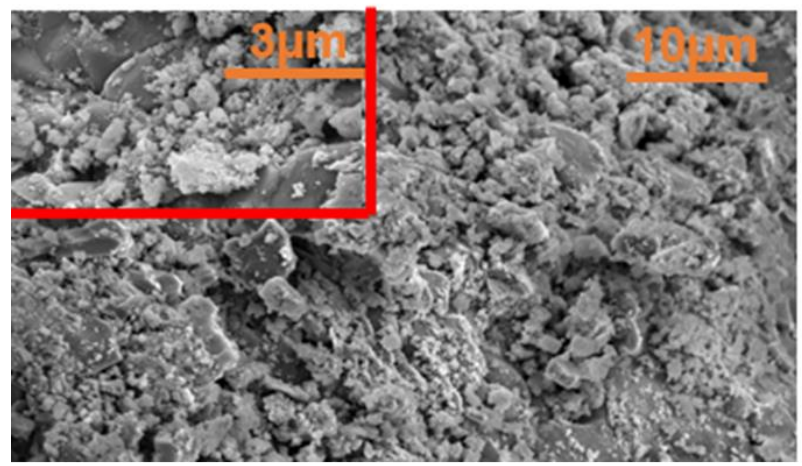

(c)

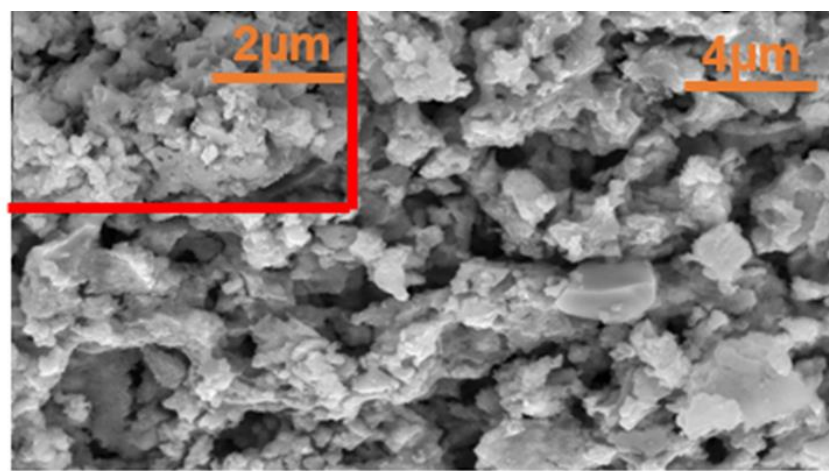

(b)

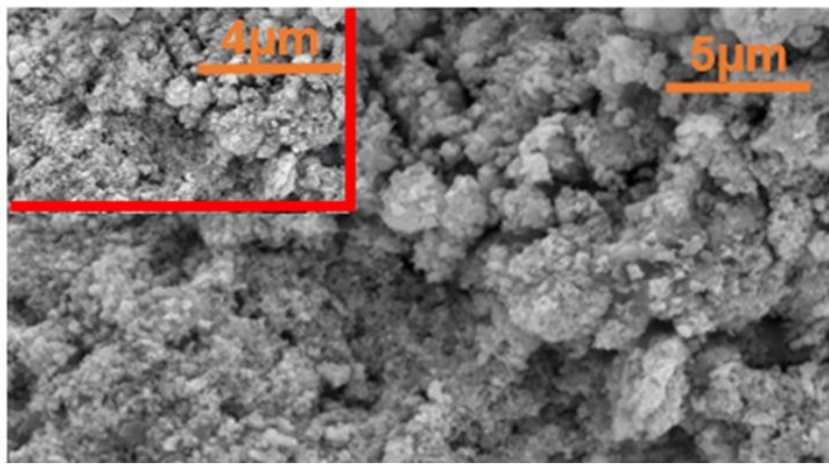

(d)

Figure SI-1: SEM images of (a) Al-F (b) Al-F-Ti-5 (c) Al-F-Ti-10 (d) Al-F-Ti-15 catalysts
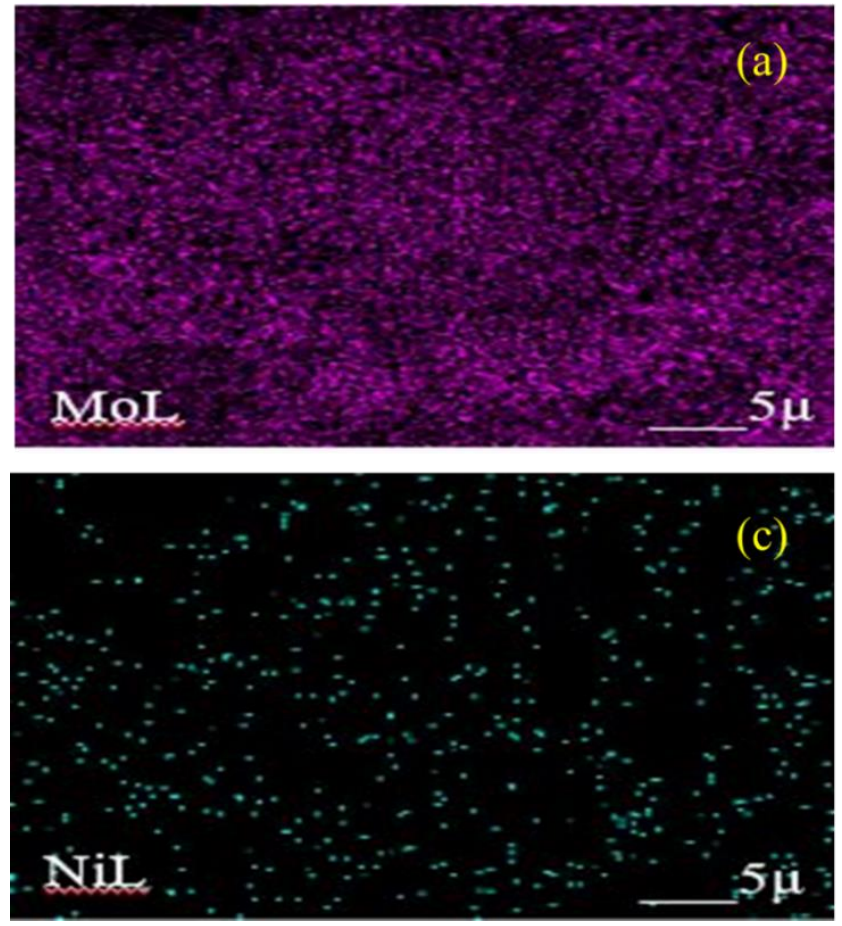
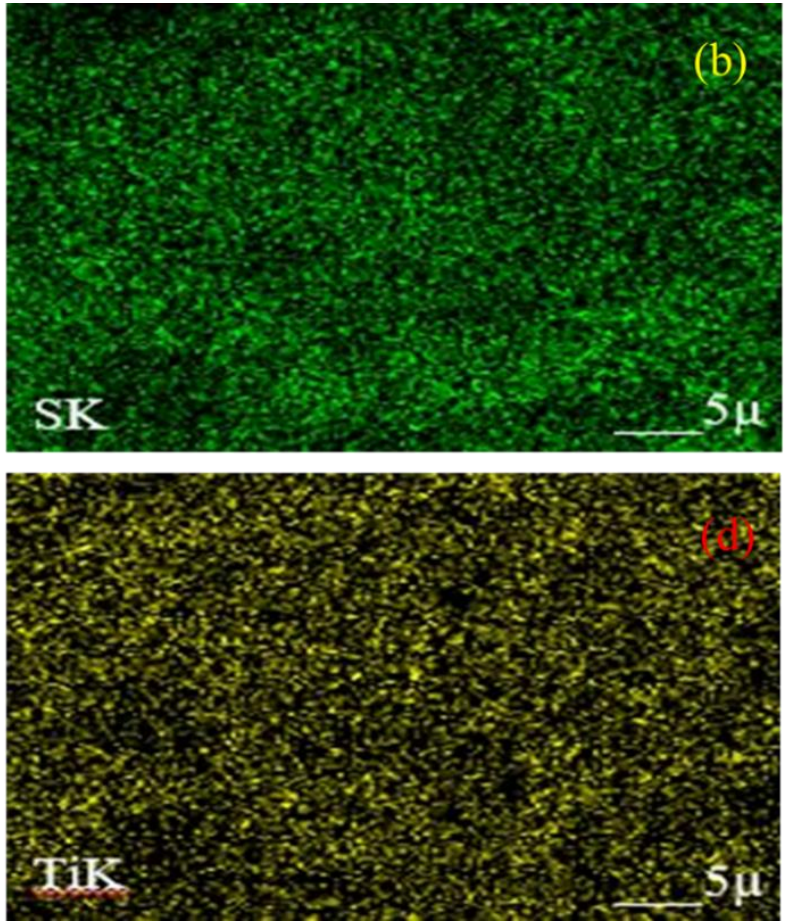
Figure SI-2: EDS mapping of Al-F-Ti-10 showing the distribution of (a) Mo (b) S (c) Ni (d) Ti in the catalyst

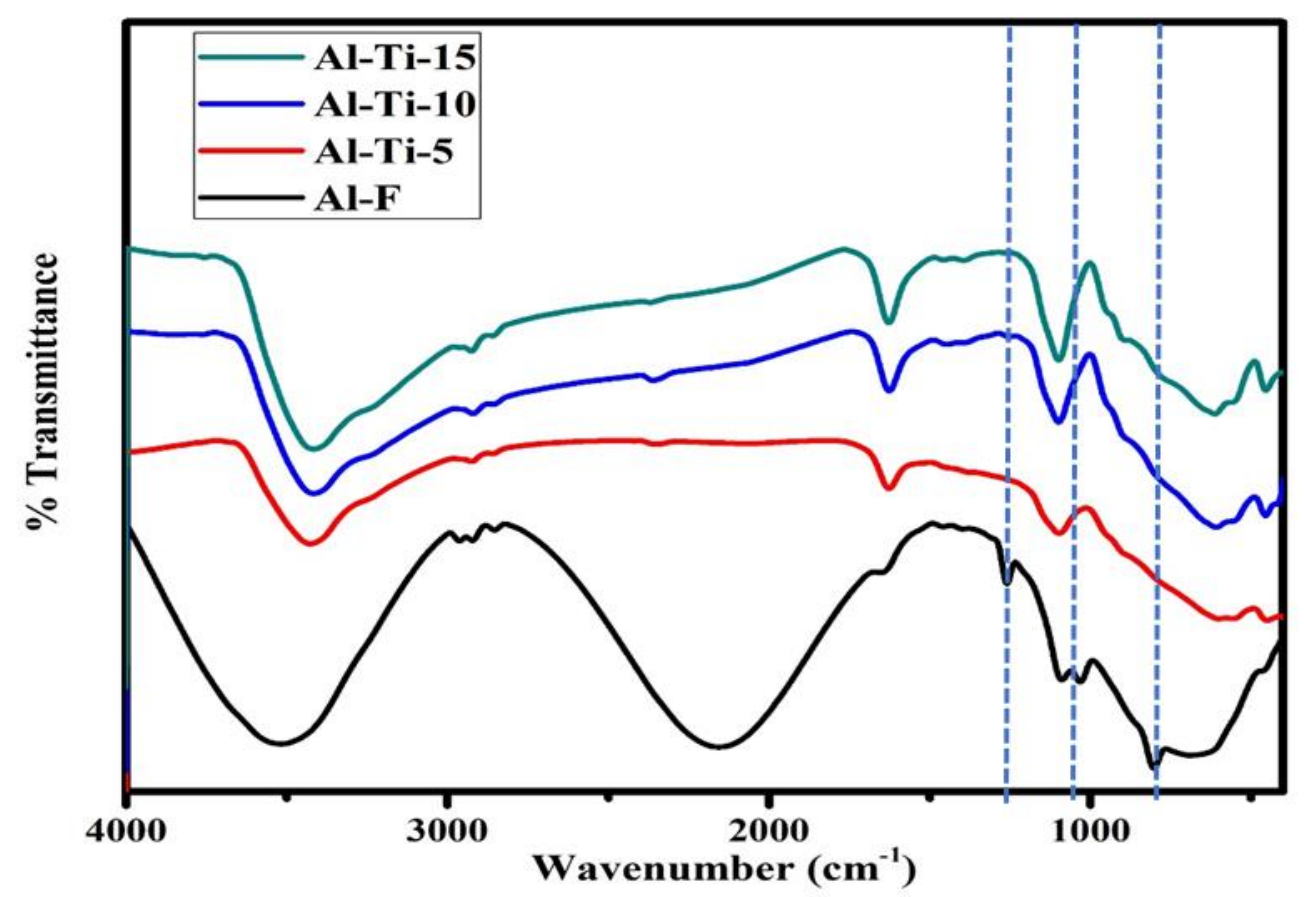

Figure SI-3: FTIR analysis of (a) Al-F (b) Al-F-Ti-5 (c) Al-F-Ti-10 (d) Al-F-Ti-15 catalysts 

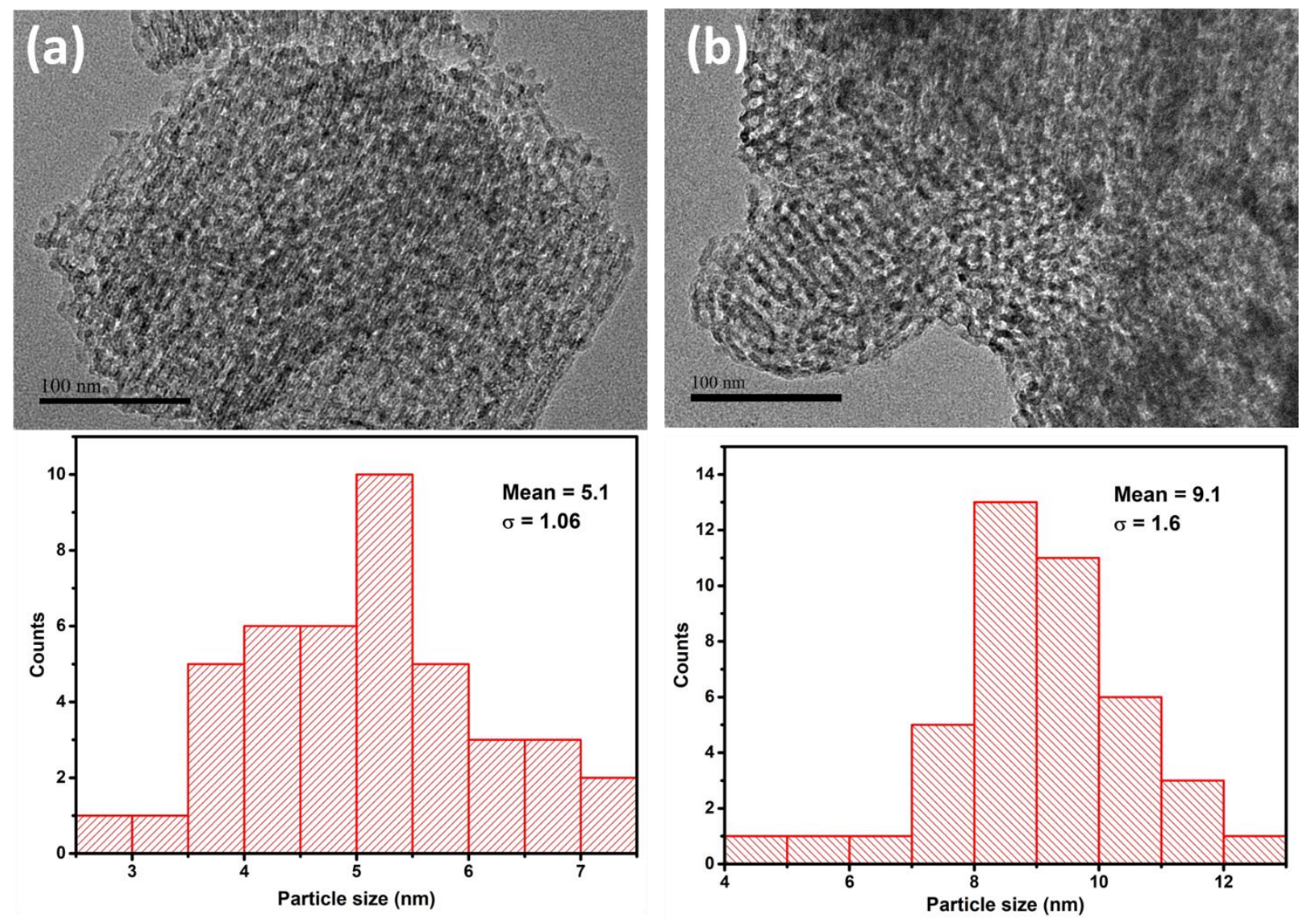

Figure SI-4: $\mathrm{MoS}_{2}$ particles size distribution of (a) Al-F and (b) Al-F-Ti-10 catalysts. 


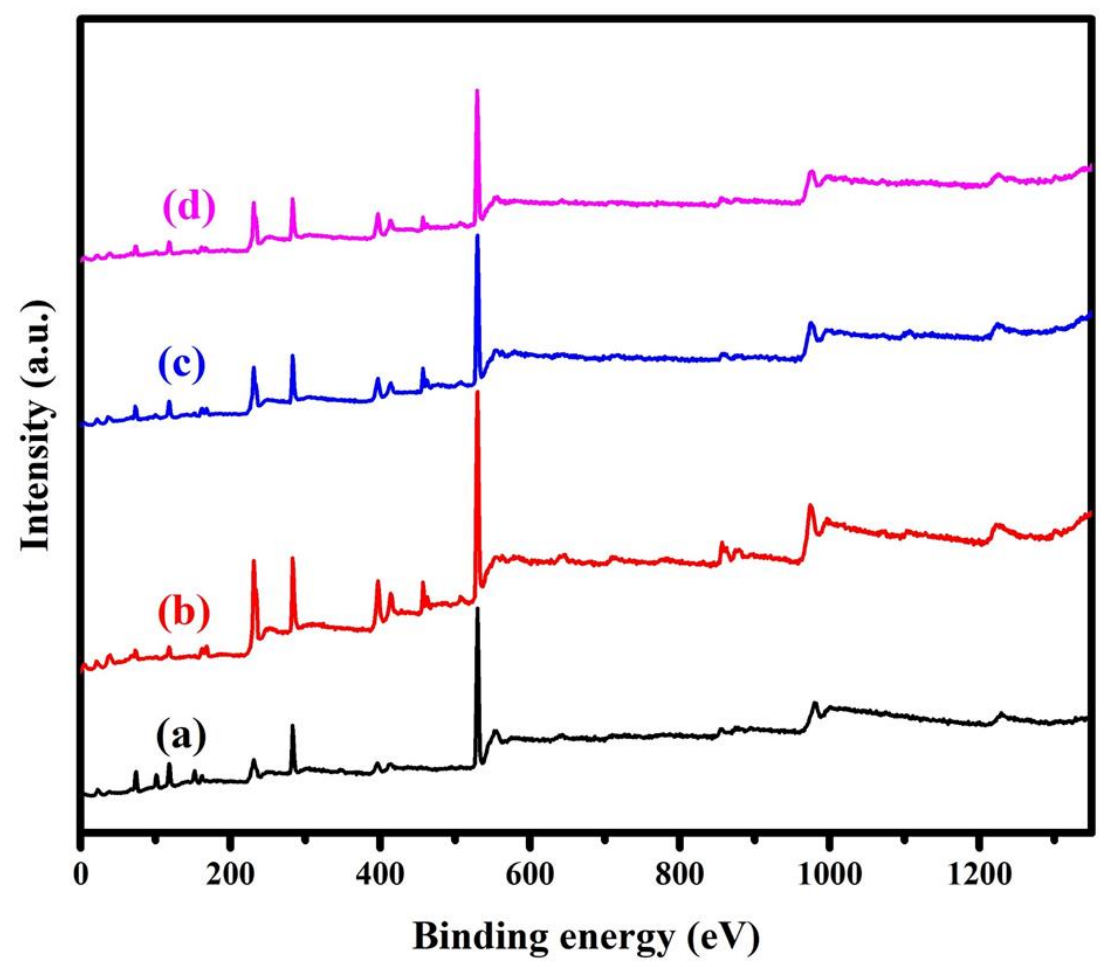

Figure SI-5: XPS survey spectra for (a) Al-F, (b) Al-F-Ti-5, (c) Al-F-Ti-10, (d) Al-F-Ti-15, showing the peaks of the respective elemental chemical states of the catalysts.

Table S1: XPS relative concentration of Mo species in the studied catalysts

\begin{tabular}{|c|c|c|c|c|c|c|}
\hline Catalysts & Name & $\begin{array}{c}\text { Peak BE } \\
(\mathrm{eV})\end{array}$ & $\begin{array}{c}\text { FWHM } \\
(\mathrm{eV})\end{array}$ & $\begin{array}{c}\text { Area }(\mathbf{P}) \\
\text { CPS.eV }\end{array}$ & Atomic \% & $\mathbf{Q}$ \\
\hline \multirow{4}{*}{ Al-F } & $\mathrm{Mo}^{6+} 3 \mathrm{~d}_{5 / 2}$ & 233 & 1.92 & 2697 & 1.26 & 1 \\
\hline & $\mathrm{Mo}^{6+} 3 \mathrm{~d}_{3 / 2}$ & 236 & 1.92 & 1862 & - & 0 \\
\hline & $\mathrm{Mo}^{4+} 3 \mathrm{~d}_{5 / 2}$ & 229 & 2.66 & 2754 & 1.28 & 1 \\
\hline & $\mathrm{Mo}^{4+} 3 \mathrm{~d}_{3 / 2}$ & 233 & 2.66 & 1901 & - & 0 \\
\hline \multirow{4}{*}{ Al-F-Ti-5 } & $\mathrm{Mo}^{6+} 3 \mathrm{~d}_{5 / 2}$ & 233 & 1.47 & 16920 & 3.81 & 1 \\
\hline & $\mathrm{Mo}^{6+} 3 \mathrm{~d}_{3 / 2}$ & 236 & 1.47 & 11681 & - & 0 \\
\hline & $\mathrm{Mo}^{4+} 3 \mathrm{~d}_{5 / 2}$ & 230 & 2.04 & 7711 & 1.55 & 1 \\
\hline & $\mathrm{Mo}^{4+} 3 \mathrm{~d}_{3 / 2}$ & 233 & 2.04 & 5323 & - & 0 \\
\hline \multirow{3}{*}{ Al-F-Ti-10 } & $\mathrm{Mo}^{6+} 3 \mathrm{~d}_{5 / 2}$ & 233 & 1.73 & 9951 & 3.11 & 1 \\
\hline & $\mathrm{Mo}^{6+} 3 \mathrm{~d}_{3 / 2}$ & 236 & 1.73 & 6870 & - & 0 \\
\hline & $\mathrm{Mo}^{4+} 3 \mathrm{~d}_{5 / 2}$ & 230 & 2.41 & 5154 & 1.61 & 1 \\
\hline
\end{tabular}




\begin{tabular}{rcccccc} 
& $\mathrm{Mo}^{4+} 3 \mathrm{~d}_{3 / 2}$ & 233 & 2.41 & 3558 & - & 0 \\
& $\mathrm{Mo}^{6+} 3 \mathrm{~d}_{5 / 2}$ & 232 & 1.53 & 8792 & 4.40 & 1 \\
& $\mathrm{Mo}^{6+} 3 \mathrm{~d}_{3 / 2}$ & 235 & 1.53 & 6070 & - & 0 \\
Al-F-Ti-15 & $\mathrm{Mo}^{4+} 3 \mathrm{~d}_{5 / 2}$ & 229 & 2.17 & 2516 & 1.26 & 1 \\
& $\mathrm{Mo}^{4+} 3 \mathrm{~d}_{3 / 2}$ & 232 & 2.17 & 1737 & - & 0 \\
\hline
\end{tabular}

Table S2: Catalyst performance results: Percent DBT removal. (Process conditions: $350{ }^{\circ} \mathrm{C} ; 5$ $\mathrm{MPa}$; DBT=1000 ppm; reaction time $=0.5-4 \mathrm{~h}$ ).

\begin{tabular}{lccccccc}
\hline \multirow{2}{*}{ Catalysts } & \multicolumn{7}{c}{ Catalytic conversion of DBT in \% } \\
\cline { 2 - 8 } & $\mathbf{0 . 5 h}$ & $\mathbf{1 h}$ & $\mathbf{1 . 5 h}$ & $\mathbf{2 h}$ & $\mathbf{2 . 5 h}$ & $\mathbf{3 h}$ & $\mathbf{4 h}$ \\
\hline $\boldsymbol{\gamma}-\mathbf{A l}_{\mathbf{2}} \mathbf{O}_{3}$ & 15 & 30 & 41 & 51 & 60 & 67 & 72 \\
$\mathbf{A l - F}$ & 28 & 40 & 49 & 62 & 68 & 74 & 81 \\
$\mathbf{A l - T i}-5$ & 35 & 44 & 59 & 73 & 80 & 86 & 88 \\
$\mathbf{A l - T i - 1 0}$ & 43 & 58 & 73 & 80 & 88 & 92 & 97 \\
Al-Ti-15 & 33 & 41 & 56 & 63 & 69 & 75 & 85 \\
\hline
\end{tabular}

Table S3: Catalyst performance results: Product Distribution (\%) after $0.5 \mathrm{~h}$ reaction time. (Process conditions: $350^{\circ} \mathrm{C} ; 5 \mathrm{MPa}$; DBT=1000 ppm).

\begin{tabular}{lcccc}
\hline Catalysts & \multicolumn{4}{c}{ Product distribution (\%) } \\
\cline { 2 - 5 } & CHB & BP & THDBT & BP/CHB \\
\hline $\boldsymbol{\gamma}-\mathrm{Al}_{\mathbf{2}} \mathbf{O}_{3}$ & 9.6 & 88.9 & 1.5 & 9.3 \\
Al-F & - & 100 & - & - \\
Al-Ti-5 & - & 100 & - & - \\
Al-Ti-10 & - & 100 & - & - \\
Al-Ti-15 & - & 100 & - & - \\
\hline
\end{tabular}

Table S4: First-order rate constants for HDS of DBT at $350{ }^{\circ} \mathrm{C}$ after $0.5 \mathrm{~h}$ reaction time.

\begin{tabular}{|c|c|c|c|c|}
\hline Catalysts & $k_{\mathrm{HDS}} \times 10^{3}\left(\min ^{-1}\right)$ & $k_{\text {DDS }} \times 10^{3}\left(\min ^{-1}\right)$ & $k_{H Y D} \times 10^{3}\left(\min ^{-1}\right)$ & KDDS/kHYD \\
\hline$\gamma-\mathrm{Al}_{2} \mathrm{O}_{3}$ & 5.4 & 4.9 & 0.5 & 9.8 \\
\hline Al-F & 11.0 & 11.0 & 0.00 & - \\
\hline Al-Ti-5 & 14.4 & 14.4 & 0.00 & - \\
\hline Al-Ti-10 & 18.7 & 18.7 & 0.00 & - \\
\hline Al-Ti-15 & 13.4 & 13.4 & 0.00 & - \\
\hline
\end{tabular}


